\section{A QUANTITATIVE AND QUALITATIVE EVALUATION OF THE INTRODUCTION OF 'SMART' SYRINGE PUMPS INTO A PAEDIATRIC INTENSIVE CARE UNIT}

${ }^{1}$ Nurain Binti Abu Hasan, ${ }^{2}$ Andrew Wignell, ${ }^{1}$ Roger Knaggs. 'University of Nottingham; ${ }^{2}$ Nottingham University Hospitals NHS Trust

\subsection{6/archdischild-2019-nppc.10}

Aim Infusion pumps are commonly used to administer medicines by intravenous infusion. Traditionally, pumps are programmed by simply entering the required number of millilitres $(\mathrm{mL})$ per hour, and there has been no mechanism whereby the pump can alert the operator to a programming error e.g. the wrong $\mathrm{mL} /$ hour rate being entered.

More recently, 'smart' infusion pumps have become available which have in-built drug libraries. For each drug, defined dosing limits are set. Users are required to reconfirm the selected infusion rate when 'soft' limits are overridden and the infusion cannot be commenced if 'hard' limits are exceeded. Soft and hard limits can also be set for the administration of boluses from continuous infusions. This study aimed to evaluate the introduction of smart syringe pumps into a UK PICU by objective assessment of compliance with drug library use, and by obtaining direct feedback from nursing staff.

Methods Data was collected over a 4 week period, immediately following the introduction of new Alaris CC smart syringe pumps. Objective assessment of drug library use utilised a piloted data collection form. One form was completed for each infusion running at the time of daily data collection. The prescription, syringe label, and programmed pump parameters were checked for each infusion and any discrepancies noted. Where the drug library was not being used, reasons for non-use were recorded. Nursing views on the benefits and potential risks of the new smart syringe pumps were determined through individual and group interviews, each following the same pre-prepared format.

Results 79 individual drug infusions were observed. 4 (5\%) were being given without using the drug library, i.e. just in terms of $\mathrm{mL} /$ hour; in all cases, the explanation was the fact that the drug was not included in the library. 73 (92\%) of the infusions observed were continuous, the remainder intermittent. 13 nurses were interviewed: the unanimous view was that the new smart pumps improved patient safety. One nurse described a situation where the smart pump had prevented a 10 -fold paracetamol overdose. Many nurses commented that pre-defined sedation boluses were a definite patient-safety benefit, both in terms of preventing incorrect dosing or the bolusing of the wrong drug. Nurses, in general, felt that the safety benefits of smart pumps applied equally to intermittent and continuous infusions. Nurses found the drug libraries easy to use, and whilst infusion set-up may take marginally longer with the drug libraries than without, this was offset by patient safety benefits. It was noted that the longer time taken to programme the pumps may diminish as familiarity increases. A small number of minor issues with the drug library were identified through nursing feedback, though none of them were patient-safety critical. This has allowed the drug library to be modified before the same pumps are also introduced to general wards across the Children's Hospital.

Conclusion The drug libraries in the new smart syringe pumps were being routinely and correctly used throughout the study period. Nursing staff had embraced the new technology, seeing clear patient safety benefits.

\section{P001 \\ CREATING ACCEPTABLE TABLETS (CAT) - A FEASIBILITY STUDY TO ASSESS THE SWALLOWABILITY AND ACCEPTABILITY OF DIFFERENT SIZED PLACEBO TABLETS IN CHILDREN AND YOUNG PEOPLE: AN INTERIM ANALYSIS}

${ }^{1}$ Louise Bracken, 'Emma McDonough, 'Fiona Wilson, 'Samantha Ashleigh, ' $U$ deme Ohia, ${ }^{2}$ Punam Mistry, ${ }^{3}$ Huw Jones, ${ }^{3}$ Nazim Kanji, ${ }^{4}$ Fang Liu, ${ }^{1}$ Matthew Peak. ${ }^{1}$ Alder Hey Children's NHS Foundation Trust; ${ }^{2}$ University of Birmingham; ${ }^{3}$ Quotient Sciences; ${ }^{4}$ University of Hertfordshire

\subsection{6/archdischild-2019-nppc.11}

Aim To determine the feasibility of investigating the swallowability and acceptability of different sized placebo tablets in children and young people (CYP) aged 4-12 years and estimate consent/recruitment rates for a future study.

Methods Participants were asked to swallow three different sized placebo tablets; $6 \mathrm{~mm}, 8 \mathrm{~mm}$ and $10 \mathrm{~mm}$ in a standardised order, smallest to largest. The recruitment target is 50 children, including both healthy children and NHS inpatients/outpatients. Following informed consent participants were shown a short video demonstrating how a tablet can be swallowed. The researcher observed and recorded the child's facial expressions as they swallowed the tablet. ${ }^{1}$ Any tablet chewing, signs of choking and/or coughing during and after swallowing were recorded. After each administration, an internal inspection of the mouth was conducted by the researcher to identify any residue or non-swallowed tablet. ${ }^{2}$ The participants assessed the swallowability and acceptability of the samples using a questionnaire that included a 5-point hedonic facial scale and voluntary feedback was provided. Faces 1-3 on the hedonic scale were deemed acceptable to the participant. The process described above was repeated for swallowing the $8 \mathrm{~mm}$ and $10 \mathrm{~mm}$ tablets. Participants were offered all three different sized placebo tablets and were given the option to stop or continue at each stage. Children who successfully swallowed all three samples were asked if they would be willing to take part in the study again if the tablet administration order was randomised rather than in order of increasing size.

Results To date, 32 participants aged between 4-12 years have taken part in the study (mean age 7.7 years). Twelve children were aged 6 or under. All 32 participants attempted to swallow the $6 \mathrm{~mm}$ tablet, 27 attempted the $8 \mathrm{~mm}$ and 25 children attempted the $10 \mathrm{~mm}$ tablet. $78.1 \%$ successfully swallowed the $6 \mathrm{~mm}$ tablet and $85.2 \%$ successfully swallowed the $8 \mathrm{~mm}$ and $10 \mathrm{~mm}$ tablets. Participants were also asked about the most important factors when taking a tablet, data were available for $30 / 32$ participants and the most important factor was deemed tablet size $(17 / 30)$, followed by taste $(7 / 30)$ responses. $87 \%$ of children who swallowed all three samples reported that they would be willing to take part in the study again if the tablet size order was randomised.

Conclusion The preliminary results show that tablets may be an acceptable dosage form for children from 4 years old. Tablet size was deemed to be the most important factor of swallowability/acceptability of tablets in children. The full data set should give further information about optimal tablet size for CYP which is currently unknown. This feasibility study will collate data on key variables which will inform the design of a larger, definitive study including the sample size based on observed recruitment/consent rates.

\section{REFERENCES}

1. Zeinstra GG, Koelen MA, Colindres $D$, et al. Facial expressions in school-aged children are a good indicator of 'dislikes', but not of 'likes'. Food Quality and Preference 2009 December:20(8):620-624. 